\title{
PRACTICE
}

- Treatment planning is an essential part of orthodontic management

- Consider the treatment aims first, then the treatment plan

- The teeth and periodontium must be healthy before starting orthodontic treatment

- To help ensure a successful treatment outcome the oral hygiene and diet must be good

- Choosing the correct appliance is important

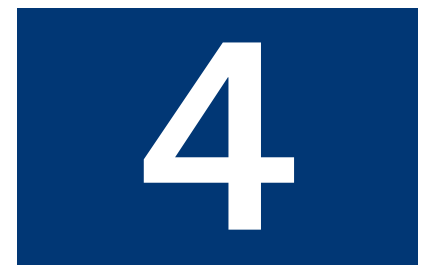

VERIFIABLE CPD PAPER

\section{Orthodontics. Part 4: Treatment planning}

\author{
D. Roberts-Harry ${ }^{1}$ and J. Sandy ${ }^{2}$
}

\section{ORTHODONTICS \\ 1. Who needs orthodontics?}

2. Patient assessment and examination I

3. Patient assessment and examination II

\section{Treatment planning}

5. Appliance choices

6. Risks in orthodontic treatment

7. Fact and fantasy in orthodontics

8. Extractions in orthodontics

9. Anchorage control and distal movement

10. Impacted teeth

11. Orthodontic tooth movement

12. Combined orthodontic treatment

\section{${ }^{1 *}$ Consultant Orthodontist, Orthodontic} Department, Leeds Dental Institute, Clarendon Way, Leeds LS2 9LU; ${ }^{2}$ Professor in Orthodontics, Division of Child Dental Health, University of Bristol Dental School, Lower Maudlin Street, Bristol BS1 2LY *Correspondence to: D. Roberts-Harry E-mail: robertsharry@btinternet.com

\section{Refereed Paper}

doi:10.1038/sj.bdj.4810820 ( ) British Dental Journal 2003; 195: 683-685

The treatment plan is an integral part of orthodontic management. It should be divided into both treatment aims (what do you want to do?) and plan (how are you going to do it?). The treatment aims will include, for example overjet reduction. The plan will consider how to create space in order to accomplish this as well as the appliance system that will be used.

Treatment planning is the second most important part of orthodontic management following the patient examination. It is helpful to divide treatment planning into two sections, treatment aims and treatment plan. Although it is possible that orthodontic treatment can influence the skeletal form when growth-modifying (functional) appliances are used, it has little effect on soft tissues, tooth size and arch length. Remember that it is not necessary to treat every malocclusion and the benefits to the patient should be carefully assessed prior to undertaking any orthodontic treatment.

\section{TREATMENT AIMS}

The following list is not comprehensive and has to be tailored to the individual case. Some of the problems that may need to be addressed during treatment are:

- Improve dental health

- Relieve crowding

- Correct the buccal occlusion

- Reduce the overbite

- Reduce the overjet

- Align the teeth

As emphasised previously, it is essential that the oral health is of a high standard before treatment starts. Carious teeth should be restored and the periodontal condition and oral hygiene should be excellent before treatment starts.

\section{Relieve crowding}

The decision to extract teeth needs to be carefully considered and depends on the degree of crowding, the difficulty of the case and the degree of overbite correction.

\section{Correct the buccal occlusion}

The key to upper arch alignment is to get the canines into a Class I relationship (Fig. 1).

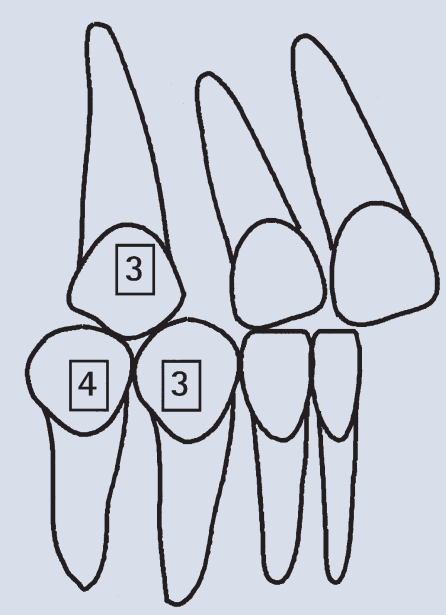

Fig. 1 It is important to achieve a Class I canine position in order to fully correct the overjet and the buccal segment relations 


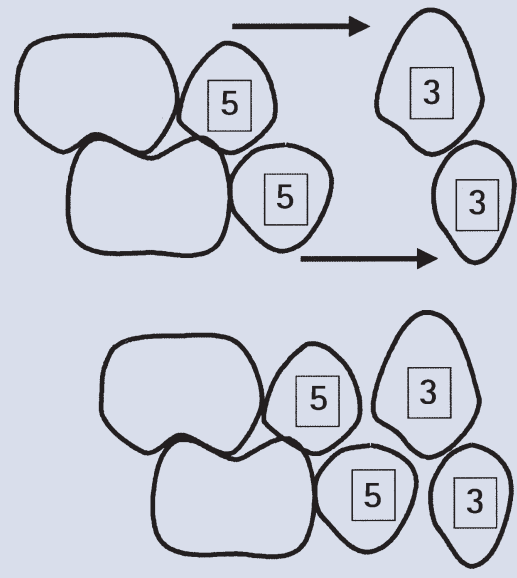

Providing the lower incisors are well aligned, achieving this will generally produce sufficient space to align the upper incisors.

In order to get the canines Class I there are, in general two choices for the molar relationship at the end of treatment; either Class I or a full unit Class II. This will be covered in more detail later in the section on treatment plan.

\section{Overbite and overjet reduction}

The overbite should always be reduced before overjet reduction is attempted. A deep overbite will physically prevent the overjet from being reduced because of contact between the upper and lower incisors.

\section{Retention}

Once the overjet has been reduced and or upper incisors have been aligned a retainer should be
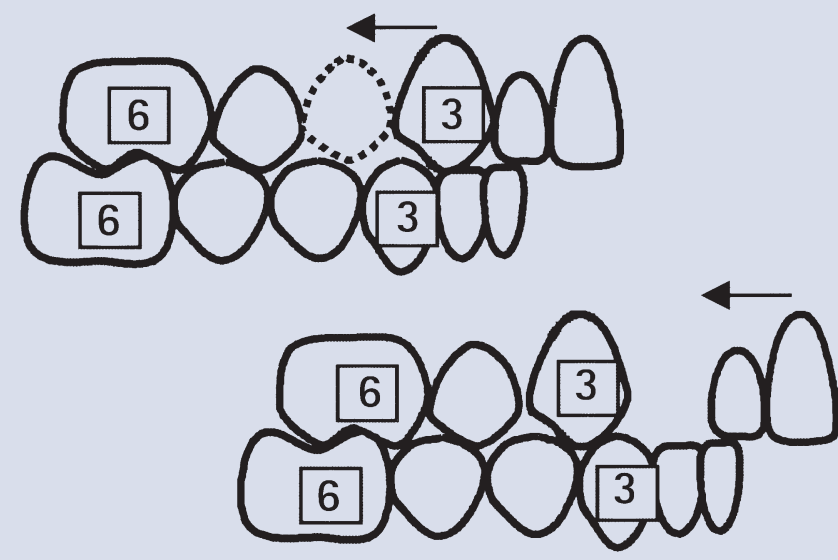

Fig. 3 Where upper premolars alone are extracted (assuming no crowding in the lower arch), reduction of the overjet and space closure means the molar relationship must be a full unit Class II fitted. These are designed to reduce the risk of relapse post treatment by allowing remodelling and consolidation of the alveolar bone around the teeth and reorganisation and maturation of the periodontal fibres. There are many different types of retainers but they are generally removable or fixed. There are no hard and fast rules regarding the length of time retention should continue. The authors recommend for removable appliance treatment that retention should continue for 3 months full time and 3 months at night-time only. For fixed appliance cases this should be 3 months full time and a minimum of 9 months at night-time only. At the end of this minimum year's worth of retention, discretionary wear should be advised. This means that the patient is given the option of discarding the retainer if they are fed up with wearing it, or continuing on a part-time regime to give the teeth the best possible chance of staying straight. If they decide to stop wearing the retainer they should be warned there is no guarantee that the teeth will remain straight throughout life and the only way to improve this prospect is by indefinite (ie life-long) wearing of the retainer.

Some cases, especially those that were spaced or where rotations were present prior to treatment, should be retained indefinitely, usually with bonded retainers.

\section{TREATMENT PLAN}

The treatment plan should be considered as follows:

- Oral health

- Lower arch

- Upper arch

- Buccal occlusion

- Choose the appliance

\section{Oral health}

Tooth brushing and diet advice must be given and written in the notes. Daily fluoride rinses are also recommended. Caries must be treated and periodontal problems appropriately addressed.

\section{Lower arch}

Plan the lower arch first. The size and form of the lower arch should generally be accepted. Excessive expansion in the buccal regions or proclination of the lower incisors is contraindicated in most cases because the soft tissues will generally return the teeth to their original position.

The need for extractions depends on the degree of crowding. In some cases, slight proclination of the lower incisors and expansion in the lower premolar region is acceptable, although this should be kept to a minimum in carefully planned cases. Generally this type of treatment is confined to the correction of mild crowding (less than $5 \mathrm{~mm}$ ), cases where incisors have been retroclined by a digit habit or trapped in the vault of the palate, or during development of Class II Division 2 malocclusions especially 
where there is a deep bite. Any case where the overbite is excessive must be very carefully assessed before extraction decisions are made.

As the degree of crowding increases from 5-10 $\mathrm{mm}$ the need for extractions increases and with more than $10 \mathrm{~mm}$ of crowding extractions are nearly always required. If spontaneous alignment or removable appliances are to be used, first premolars are usually the extraction of choice because they are near to the site of crowding, allow the canines to upright and produce the best contact point relationship. If other teeth are to be extracted then generally fixed appliances will be required. Crowding tends to worsen with age and is thought to be related to facial growth which continues at least until the fifth decade.

\section{Upper arch}

Plan the upper arch around the lower. If extractions are undertaken in the lower arch these should generally be matched by extractions in the upper. If no extractions are carried out in the lower arch the space for upper arch alignment may come from either distal movement of the upper buccal segments or extraction of upper premolars. The choice depends on the space requirements and the buccal occlusion. As the degree of crowding and overjet increase, then the space requirements will also increase and it is more likely that extractions as opposed to distal movement will be indicated.

Determine whether the teeth are favourably positioned for spontaneous alignment. If appliances are needed can removable or fixed appliances accomplish the tooth movements?

\section{Plan the buccal occlusion}

Consider whether this needs to be corrected and if so how. If headgear is to be used, should it be used in conjunction with a removable or a fixed appliance? If the lower arch is crowded, space may be created by the removal of two lower premolars. This is then matched by upper premolar extractions and the molar relationship must be Class I at the end of treatment to allow the arches to fit together (Fig. 2).

However if the lower arch is well aligned, space to align the upper arch can be created by either upper premolar extractions or by distal movement of the upper buccal segments. The choice depends on how much space is required and what the molar relationship is at the start of treatment. Generally the more Class II the molars are the more likely one will opt for premolar extraction rather than distal movement. Moving molars more than $3-4 \mathrm{~mm}$ distally is

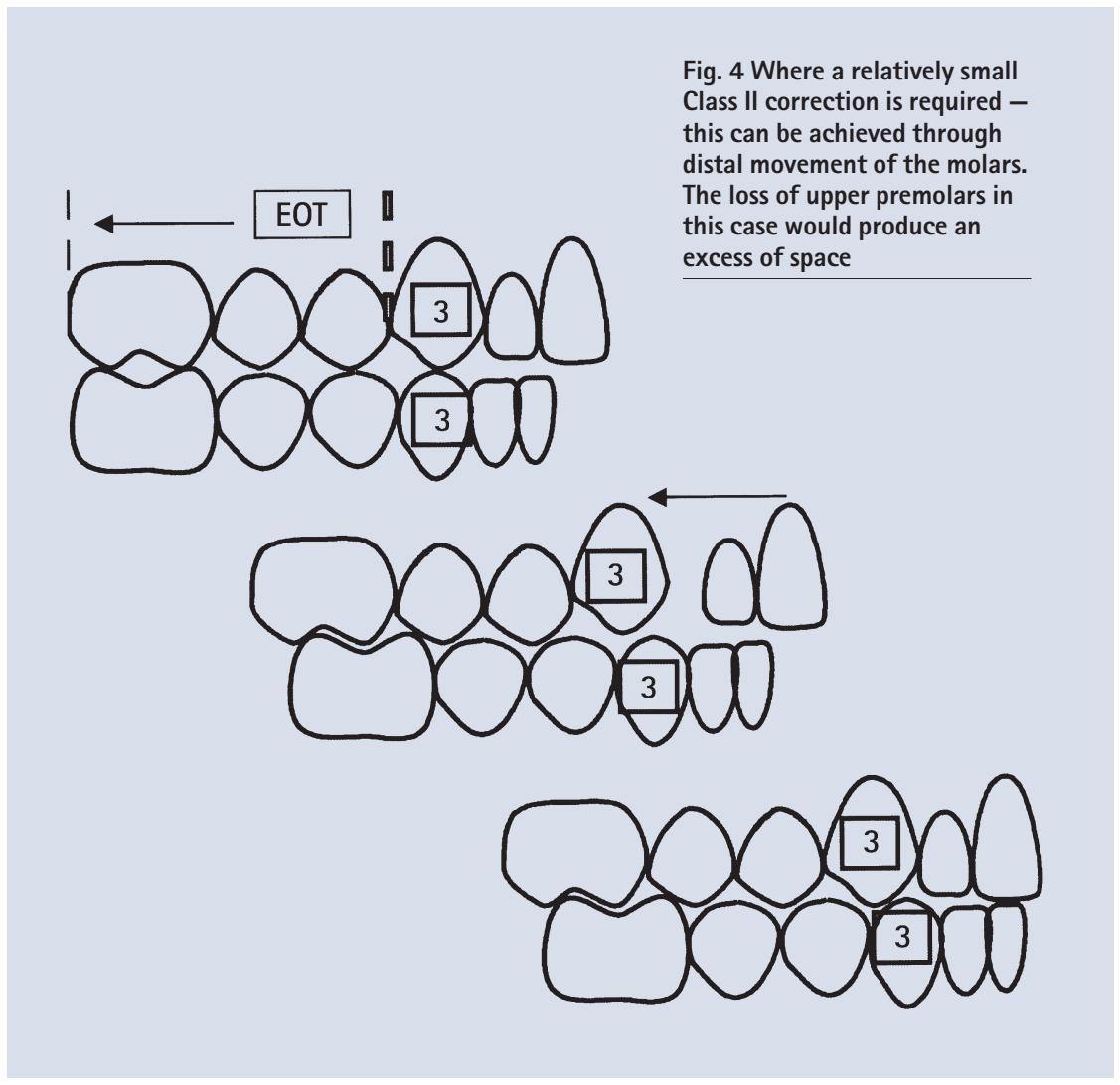

possible but becomes increasingly demanding on patient co-operation. In circumstances where the space requirements are large, upper premolar extraction reduces the treatment time and increases patient compliance. Figure 3 shows the sequence of events when upper premolar extraction alone is undertaken as an aid to overjet reduction.

The nearer to Class I the initial buccal occlusion is, the more likely it will be that distal movement is appropriate. Therefore, space requirements that involve less than half a unit Class II correction can be accomplished by distal movement of the molars in a relatively short time with more chance of good patient co-operation (Fig. 4). Extracting upper premolars in these cases produces an excess of space and may increase the treatment time.

\section{Choose the appliance}

Once the need for extractions has been considered the appropriate appliance should be selected. This can involve allowing some spontaneous alignment to occur, using removable, fixed or functional appliances with the addition of extraoral traction or anchorage. Appliance choices are covered in the next section. 